ARTICLE

https://doi.org/10.1038/s41467-019-10860-3

\title{
MFS transporter from Botrytis cinerea provides tolerance to glucosinolate-breakdown products and is required for pathogenicity
}

David Vela-Corcía (10 ${ }^{1}$, Dhruv Aditya Srivastava ${ }^{1}$, Avis Dafa-Berger ${ }^{1}$, Neta Rotem ${ }^{1}$, Omer Barda $^{1} \&$ Maggie Levy $^{1}$

Glucosinolates accumulate mainly in cruciferous plants and their hydrolysis-derived products play important roles in plant resistance against pathogens. The pathogen Botrytis cinerea has variable sensitivity to glucosinolates, but the mechanisms by which it responds to them are mostly unknown. Exposure of $B$. cinerea to glucosinolate-breakdown products induces expression of the Major Facilitator Superfamily transporter, $m f s G$, which functions in fungitoxic compound efflux. Inoculation of B. cinerea on wild-type Arabidopsis thaliana plants induces $\mathrm{mfs} G$ expression to higher levels than on glucosinolate-deficient $A$. thaliana mutants. A B. cinerea strain lacking functional $m f s G$ transporter is deficient in efflux ability. It accumulates more isothiocyanates (ITCs) and is therefore more sensitive to this compound in vitro; it is also less virulent to glucosinolates-containing plants. Moreover, $\mathrm{mfs} G$ mediates ITC efflux in Saccharomyces cerevisiae cells, thereby conferring tolerance to ITCs in the yeast. These findings suggest that $\mathrm{mfs} G$ transporter is a virulence factor that increases tolerance to glucosinolates.

\footnotetext{
${ }^{1}$ Department of Plant Pathology and Microbiology, the Robert H. Smith Faculty of Agriculture, Food and Environment, The Hebrew University of Jerusalem, Rehovot 76100, Israel. Correspondence and requests for materials should be addressed to M.L. (email: maggie.levy@mail.huji.ac.il)
} 
O ne of the key plant defense mechanisms against pathogenic microorganisms relies on the production of phytoalexins ${ }^{1-3}$ and phytoanticipins ${ }^{4,5}$, such as the aminoacid-derived metabolites camalexin and glucosinolate (GS), respectively ${ }^{6}$. Fungal pathogens use various pathogenicity and virulence strategies to efficiently detoxify or eliminate these phytoanticipins and phytoalexins $s^{5,7,8}$. Detoxification can be achieved by enzymatic conversion of phytoanticipins and phytoalexins in many phytopathogenic fungi, whereas elimination can be achieved by active efflux, as shown, for example, in Botyrytis cinerea for the phytoalexins resveratrol and eugenol ${ }^{7-11}$.

In several fungal species, active efflux by ATP-binding cassette (ABC) or major facilitator superfamily (MFS) transporters provides resistance to various toxic compounds, including not only secondary metabolites but also antibiotics and fungicides ${ }^{12-14}$. Although crucial to pathogenicity on plants, only a limited number of $\mathrm{ABC}$ transporters have been studied for their role in pathogenesis, for example, Magnaporthe grisea $\mathrm{ABC} 1, \mathrm{ABC} 3$ and $\mathrm{ABC} 4$ on rice ${ }^{15-17}, B$. cinerea atrB on grapevine and Arabidopsis thaliana ${ }^{10,14}$, Gibberella pulicaris $\mathrm{ABC} 1$ on potato ${ }^{18}$, and Mycosphaerella graminicola atr4 on wheat ${ }^{19}$.

It is important to note that $\mathrm{ABC}$ and MFS transporters are not limited to plant pathogens; their active efflux is used by many other organisms as well ${ }^{20}$. In the human pathogens Candida and Aspergillus spp., for example, these transporters contribute to resistance to azole fungicide, making therapy less efficient ${ }^{21}$. The ABC transporter AFR1 of the human pathogen Cryptococcus neoformans provides defense against fluconazole and acts as a virulence factor by protecting the fungi from toxic compounds produced by the host phagocytes ${ }^{22}$. Probably one of the beststudied bacterial ABC transporters is LmrA of Lactobacillus lactis, which contributes to bacterial antibiotic resistance in humans ${ }^{23,24}$.

We recently showed that $B$. cinerea displays variable sensitivity to GSs and their degradation products, whereas Alternaria brassicicola, a specialist Brassica pathogen, was more tolerant to GS and its hydrolysis products ${ }^{25}$. Although the toxicity of isothiocyanates (ITCs) and other GS-hydrolysis products to a range of fungi has been demonstrated, the mechanism of toxicity and the fungi's ability to tolerate or detoxify GS-hydrolysis products are still largely obscure.

B. cinerea is a necrotrophic plant pathogen with a broad host range ( $>200$ plant hosts), including the agriculturally important cruciferous crops. It is the causal agent of gray mold, resulting in enormous economic losses, both during plant growth and in the postharvest phase ${ }^{26,27}$. B. cinerea control depends on repeated use of chemical fungicides, which causes rapid development of fungicide resistance ${ }^{28}$. Thus, understanding the mechanisms by which this pathogen detoxifies its host defense system is important for revealing basic cellular processes and crucial for the development of new strategies to control this pathogen.

Here, we report on the identification of a $B$. cinerea MFS transporter, designated $m f_{s} G$, involved in detoxifying ITCs. This transporter was differentially expressed in the presence of different ITCs in vitro, and was upregulated in planta during interaction with wild-type $A$. thaliana. A $B$. cinerea isolate with $m f_{s} G$ deletion $\left(\Delta m f_{s} G\right)$ was less able to tolerate ITCs in vitro, and therefore showed reduced virulence toward $A$. thaliana. B. cinerea $m f_{s} G$ enabled tolerance to benzyl ITC (BITC) in the heterologous Saccharomyces cerevisiae system, further supporting our findings that $m f_{s} G$ functions in the efflux of GS-breakdown products during plant-pathogen interactions.

\section{Results}

ITCs inhibit mycelial growth of $\boldsymbol{B}$. cinerea in vitro. To determine the inhibitory effect of the defense-related GS-breakdown
products-ITCs-on B. cinerea (isolate B05.10), radial growth was tested in the presence of different concentrations of propyl ITC (PITC), BITC, and 2-phenethyl ITC (PhITC). Both BITC and PhITC inhibited radial growth in a dose-dependent manner (Fig. 1a), whereas the inhibitory effect of PITC was minor (Fig. 1a). B. cinerea was most sensitive to BITC, with complete (100\%) inhibition at $400 \mu \mathrm{M}$. In comparison, only $70 \%$ inhibition was observed with $400 \mu \mathrm{M}$ PhITC (Fig. 1a).

Identification of a MFS gene whose expression is induced by ITCs. RNA-Seq analysis of the GS-breakdown product BITC revealed 16 differentially expressed MFS genes; only one of them was strongly upregulated and was designated $m f_{s} G$ (unpublished data; Supplementary Fig. 1).

Expression of $\boldsymbol{m f s} \boldsymbol{s}$ is induced by ITCs. Quantitative real-time PCR (qRT-PCR) analysis, using different concentrations of BITC and PhITC, revealed $m f_{s} G$ upregulation in response to BITC (Fig. 1b). The expression level of $m f s G$ was dependent on BITC and PhITC concentration. Increasing concentrations of BITC increased the relative quantity of mRNA copies of $m f_{s} G$, with a peak at $300 \mu \mathrm{m}$ BITC. Above this concentration, B. cinerea was unable to grow and relative quantification of mRNA could not be determined. Corresponding to the growth-inhibition results, $m f_{s} G$ expression was lower in the presence of PITC and PhITC compared with BITC (Fig. 1b).

$m f s G$-knockout mutants are impaired in ITC efflux and inhibited by ITCs in vitro. Knockout mutants of $m f_{s} G\left(\Delta m f_{s} G-1\right.$ and $\Delta m f_{s} G_{-2}$ ) did not exhibit $m f_{s} G$ expression (Fig. 2a). As expected, these mutants' growth was already restricted at the low BITC concentration (10 $\mu$ m; Supplementary Fig. 2). After $24 \mathrm{~h}$, the $\Delta m f s G s$ were inhibited by $65 \%$ on $200 \mu \mathrm{M}$ BITC, whereas the wild-type strain was only inhibited by $20 \%$ (Fig. 2b). Complementation of $\Delta m f_{s} G$ with the full-length $m f_{s} G$ in two strains, Comp1 and Comp2, restored $m f s G$ expression (Fig. 2a) and significantly reduced growth inhibition by BITC as compared with the knockout lines, to levels comparable to the wild-type strain (Fig. 2b). Furthermore, we demonstrated the $\Delta m f_{s} G$ hyphae are impaired in ITC efflux and accumulate four times more fluorescent ITC (FITC) than wild-type B. cinerea (Fig. 3).

$m f s G$ expression is modulated by ITC content in planta. To study the expression levels of $m f_{s} G$ in planta, we used different $A$. thaliana genotypes with different GS levels: IQD1-overexpressing plants $\left(I Q D 1^{O E}\right)$ which contain double the amount of $\mathrm{GS}^{29} \mathrm{com}$ pared with the wild type, the double-mutant cyp79B2/cyp79B3 (cyp79B2/B3) that accumulates only a quarter of the wild-type amount of $\mathrm{GS}^{30}$ and double-mutant $\operatorname{tgg} 1-3 / \operatorname{tgg} 2-1(\operatorname{tgg} 1 / 2)$ that contains wild-type GS levels but is impaired in both myrosinase hydrolytic enzymes ${ }^{31}$. The mutant and IQD1OE plants were inoculated with $B$. cinerea, and $m f s G$ expression levels were determined $48 \mathrm{~h}$ post inoculation as compared with wild-type plants. As expected, $m f_{s} G$ transporter was upregulated in plants containing high GS levels (IQD1OE), whereas it was downregulated in plants with low GS levels (cyp79B2/B3) and in plants that do not accumulate GS-breakdown products (tgg1/2) (Fig. 4a).

$\Delta m f_{s} G$ mutants are less virulent in planta. To determine the virulence of $\Delta m f_{s} G$ mutants in response to GS levels in planta, we performed pathogenicity tests of wild-type $B$. cinerea, its knockout mutant and complemented mutant on the GS-overexpressing and mutant $A$. thaliana genotypes. $B$. cinerea $\Delta m f_{s} G$ mutants were $80 \%$ less virulent on wild-type $A$. thaliana plants compared with the wild-type $B$. cinerea, as determined by measuring the 

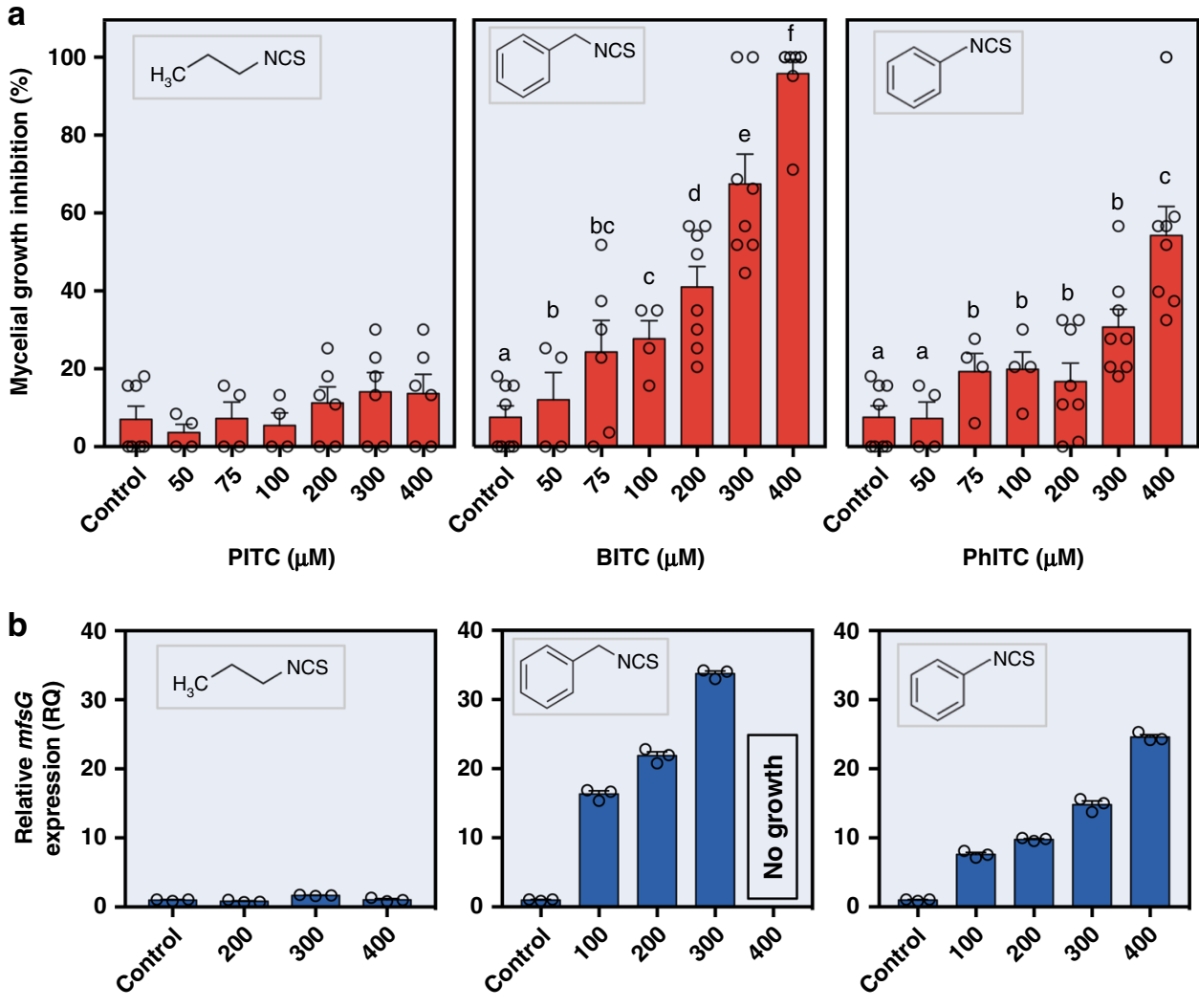

$\operatorname{PITC}(\mu \mathrm{M})$

$\operatorname{BITC}(\mu \mathrm{M})$

$\operatorname{PhITC}(\mu \mathrm{M})$

Fig. 1 Growth inhibition of $B$. cinerea and $m f s G$ expression in response to ITCs. a Diameter of $B$. cinerea colonies was measured $48 \mathrm{~h}$ post inoculation on PDA plates supplemented with different concentrations of PITC, BITC or PhITC. Growth inhibition was calculated as percentage of colony diameter of $B$. cinerea grown on PDA with no ITCs. Data are displayed as mean values $( \pm \mathrm{SE}), n>4$. Different letters above the columns indicate significant difference at $P<0.05$, as determined by Tukey-Kramer HSD test. $\mathbf{b}$ Expression of $m f s G ~ 72 \mathrm{~h}$ after exposure to different concentrations of PITC (left), BITC (middle) or PhITC (right), as determined by GRT-PCR, relative to expression on PDA without ITCs. $n=3$. Source data are provided as a Source Data file

lesion sizes $72 \mathrm{~h}$ post inoculation (Fig. $4 \mathrm{~b}$ ). The $\Delta m f_{s} G$ mutants did not produce any significant lesions on plant wild-type plants, or $\operatorname{tg} 1 / 2$ or IQD1 ${ }^{\mathrm{OE}}$ plants, all compose normal or high levels of GS. However, they produced lesions with sizes comparable to those produced by the $B$. cinerea wild-type strain on the doublemutant cyp79B2/B3, which has reduced GSs levels (Fig. 4b). Disease severity reached $B$. cinerea wild-type levels when infecting the $A$. thaliana wild type, $\operatorname{tgg} 1 / 2$ and $I Q D 1^{\mathrm{OE}}$ with the $B$. cinerea complementants of $m f s G$ (Fig. $4 \mathrm{~b}$ ).

$\Delta m f_{s} G$ mutants demonstrate lower frequency of double germ tubes. To evaluate the fitness of the wild-type, knockout mutant and complemented $B$. cinerea, the percentages of conidial germination and double germ-tube production were evaluated by microscopy. The germination rate of all strains was close to $100 \%$ with no significant differences among them. However, $\Delta m f_{s} G$ mutants showed a significantly lower percentage of double germ tubes than wild-type strain on all A. thaliana genotypes. After mutant complementation, the number of double germ tubes was comparable to the wild type (Fig. 5). The highest number of double germ tubes was observed in $c y p 79 B 2 / B 3$ and $\operatorname{tgg} 1 / 2$, and the lowest, albeit not significantly, in $I Q D 1^{\mathrm{OE}}$, which had the highest GS content (Fig. 5). Furthermore, in vitro analysis with $10 \mu \mathrm{M}$ BITC also demonstrated less double germ tubes in $\Delta m f_{s} G$ compared with the wild type (Supplementary Fig. 3).

$m f_{s} G$ confers tolerance to BITC in yeast. To further study the functionality of $m f_{s} G$, we determined its ability to induce tolerance to BITC in yeast cells. Wild-type yeast cells were highly susceptible to BITC; their growth was suppressed in the presence of $15 \mu \mathrm{M}$ BITC, as determined by the optical density of the live culture and the number of colony-forming units (Fig. 6a). Interestingly, yeast cells expressing $B$. cinerea $m f s G$ (WT::BcmfsG) were highly resistant to BITC (Fig. 6a). This was also evident in the transformed yeast cells' ability to reach their exponential stage $10 \mathrm{~h}$ earlier than the wild-type cells under exposure to BITC (Supplementary Fig. 4). Furthermore, WT::Bcmfs $G$ yeast cells accumulated 40\% less FITC than the wild-type yeast cells (Fig. 6b, c).

Structural analysis of $\boldsymbol{m} \boldsymbol{f}_{\boldsymbol{s}} \boldsymbol{G}$-encoded protein and BITC-binding analysis. $B$. cinerea $m f s G$ protein encodes 431 amino acids with predicted molecular mass of $46 \mathrm{kDa}$ and 12 putative transmembrane domains (Fig. 7a and Supplementary Fig. 5). Threedimensional structure modeling of $m f_{s} G$ protein using the Phyre2 software obtained a $100 \%$ confidence alignment with a template for MFS general substrate transporters ${ }^{32}$. When $m f s G$ structure was viewed from the side of the membrane, and rotated through $90^{\circ}$, it suggested that the conserved 12 transmembrane (TM) a-helix fold is arranged into two 6 TM helix bundles, forming a cavity at the interface between them that is accessible to either the cytoplasm or extracellular region, depending on the conformational state (Fig. 7a, Supplementary Fig. 5). When looking at the tertiary $m f_{s} G$ structure's hydrophobic interactions, we observed a hydrophobic area inside the membrane, whereas the hydrophilic area was outside, either on the cell surface or inside the cell (Fig. 8c). We further performed molecular docking to map the interactions between the BITC molecule and $m f_{s} G$ to identify a putative binding site for this compound. This putative 
a

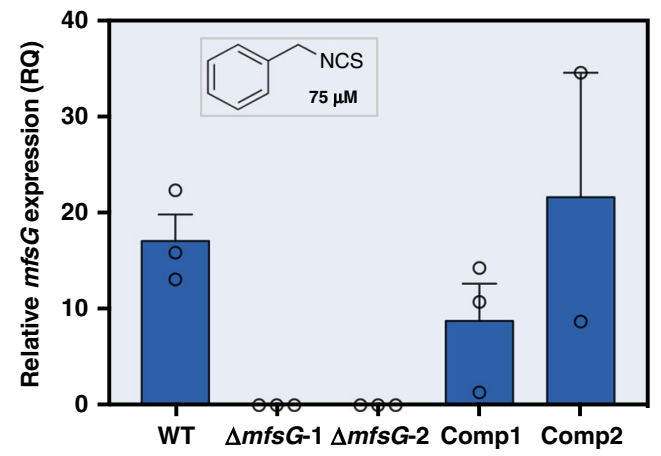

b

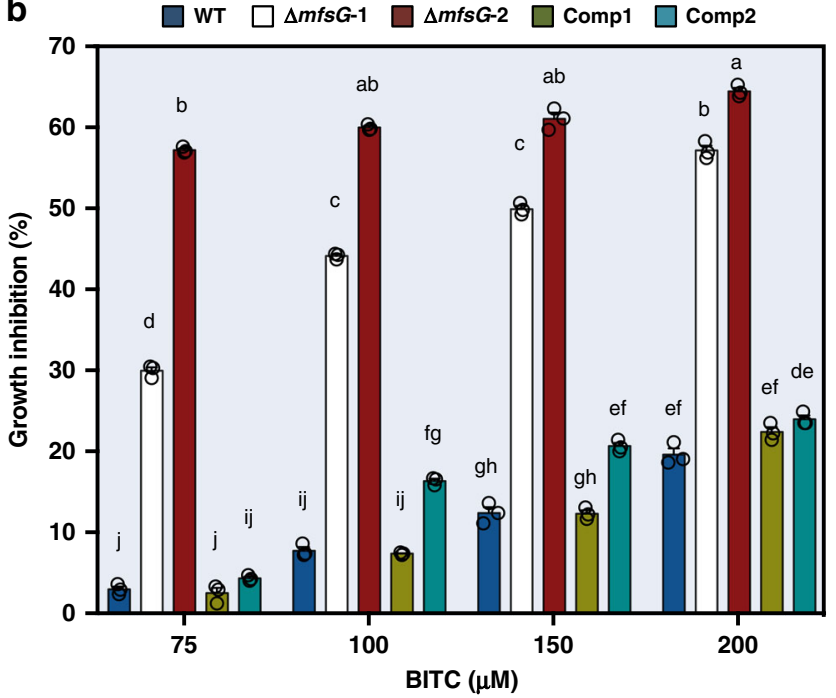

Fig. 2 Characterization of $\Delta m f s G$ mutants and complemented mutants. a Expression analysis of mutants and complemented mutants $72 \mathrm{~h}$ after exposure to $75 \mu \mathrm{M}$ BITC, $n=3$. b In vitro growth inhibition $72 \mathrm{~h}$ after exposure to different concentrations of BITC. Growth inhibition was calculated as percentage of colony diameter of wild-type (WT) B. cinerea grown on PDA with no ITCs. Data are displayed as mean values $( \pm \mathrm{SE})$. Different letters above the columns indicate significant difference at $P<$ 0.05 , as determined by Kruskal-Wallis test, $n=3$. Source data are provided as a Source Data file

binding site was formed by residues located in the internal channel of $m f_{s} G$. The proposed binding region was formed by the aromatic rings of residues Trp54, Phe254, Gln344, and Phe369 (Fig. 7b). Distances were measured between the BITC molecule and the side chains of these residues: the biggest distance was $4.175 \AA$ with Phe254, and it was bound by one hydrogen bond to Trp54 (0.86 Å) (Fig. 7c). Molecular docking was also performed using PITC and PhITC (Supplementary Fig. 6a, b). PITC was bound by one hydrogen bond to Trp54 (2.63 $)$, whereas PhITC was bound by one hydrogen bond to $\operatorname{Arg} 290(2.82 \AA)$, both in the central channel of $m f_{s} G$. Molecular docking using FITC showed that it is bound by one hydrogen bond to Trp54 (2.325 $\AA$ ) in $m f s G$ (Supplementary Fig. 6c). The thermodynamic parameters, Full fitness and free energy $(\Delta G)$, provided by SwissDock clearly indicated that the ITCs can bind tightly to $m f s G$ (Table 1). Furthermore, we demonstrated molecular docking of other GS-breakdown products, such as nitrile molecules, to $m f_{s} G$. Although 5-(methylsulfanyl)pentanenitrile bound to $m f_{s} G$ by two hydrogen bonds, one to $\operatorname{Trp} 54(2.08 \AA$ ) and the other to $\operatorname{Arg} 290$ (2.47 $\AA$ ) (Supplementary Fig. $6 \mathrm{~d}$ ), benzonitrile was bound by one hydrogen bond to Trp54 (2.25 $\AA$ ) (Supplementary Fig. 6e), but the high free energy needed to form the hydrogen bond suggested a strongly a
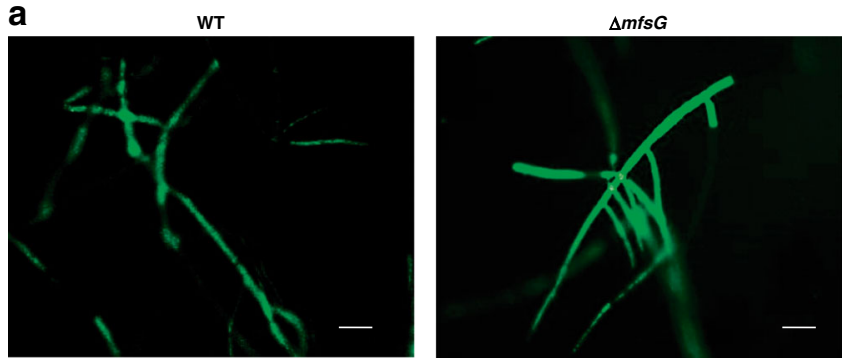

b 6.0

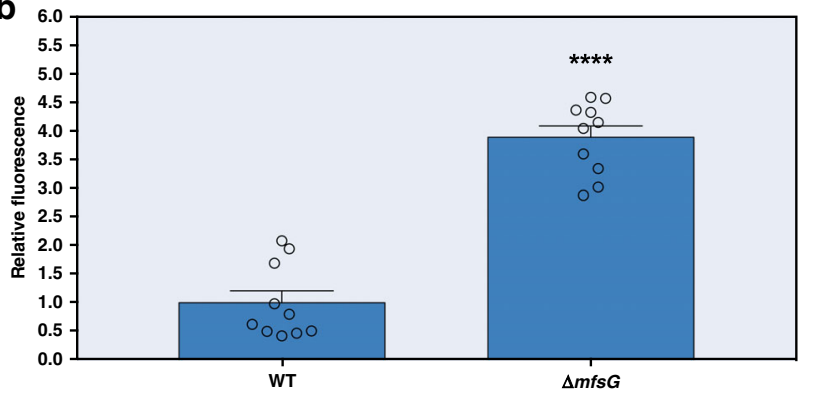

Fig. $3 \mathrm{mfs} G$ confers ITC efflux in $B$. cinerea. a Fluorescence accumulation in $\Delta m f s$ relative to the wild type (WT) 4 days after exposure to $25 \mathrm{mg} \mathrm{ml}^{-1}$ FITC. $\mathbf{b}$ Quantification of fluorescence accumulation in $\triangle m f s G$ relative to WT hyphae. Data are displayed as mean values $( \pm S E)$ of relative fluorescence. Asterisk denotes significant difference by Student's $t$ test $(P<0.001), n=10$. Scale bars are $20 \mu \mathrm{m}$. Source data are provided as a Source Data file

non-spontaneous binding process for both compounds (Table 1). Molecular docking with benzyl cyanide, another breakdown product of GS, revealed no binding sites to $m f_{s} G$ that also supported by the high free energy estimated (Table 1). A phylogenetic analysis using MFS proteins that share over $40 \%$ similarity to $m f s G$ was performed (Fig. 8a; Supplementary Table 1). Sequence alignment revealed the presence of two well-represented clusters in the neighbor-joining phylogram. The first cluster contained $m f s G$ and a closely related protein from $B$. cinerea $\mathrm{BcDW} 1$, a putative riboflavin transporter, as well as other closely related proteins. The analysis showed the existence of two other $m f s$-like proteins in B. cinerea B05.10 (gene IDs: BCIN_03g02910 and BCIN_02g05130), belonging to a separate cluster of $m f s G$, with homologs in B. cinerea T4, B. cinerea BcDW1 and their closest relative Sclerotinia sclerotiorum. Structural comparison of these three protein sequences from $B$. cinerea B05.10 showed that they have characteristics of MFS motifs and transmembrane domains in common (Fig. 8b, d), but they clearly differ in many residues along the amino-acid sequence, such as the aromatic residues that compose the core channel of $m f s G$; one of the missing residues was Phe254, which seems to be involved in GS-derivative binding to $m f s G$, as predicted by the molecular docking simulation. Nevertheless, thermodynamic analysis demonstrates lower possibility for binding (Supplementary Table 2) and no binding sites were found when molecular docking was carried out using BITC and $m f s$-like proteins from B. cinerea B05.10, T4 and S. sclerotiorum. This suggests that $B$. cinerea B05.10 $m f s G$ is specific for GS-derivative detoxification ${ }^{33-36}$.

\section{Discussion}

Many studies have shown that the interaction between fungal pathogens and their host plant is mediated by secondary metabolites and corresponding pathogen tolerance. However, those studies focused mainly on the mechanisms of secondary metabolite detoxification and little data, if any, have been accumulated 
a

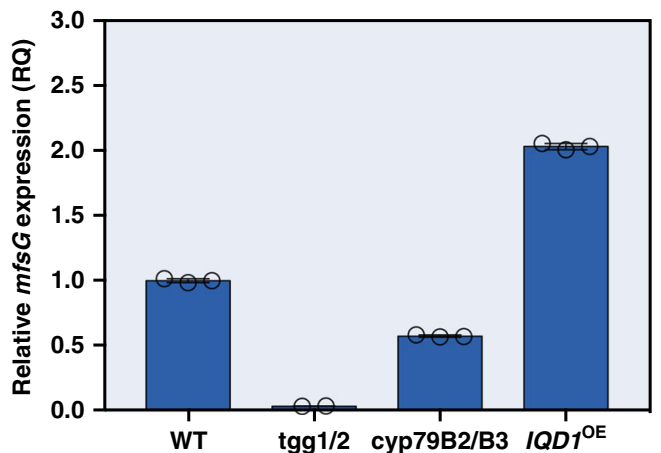

b
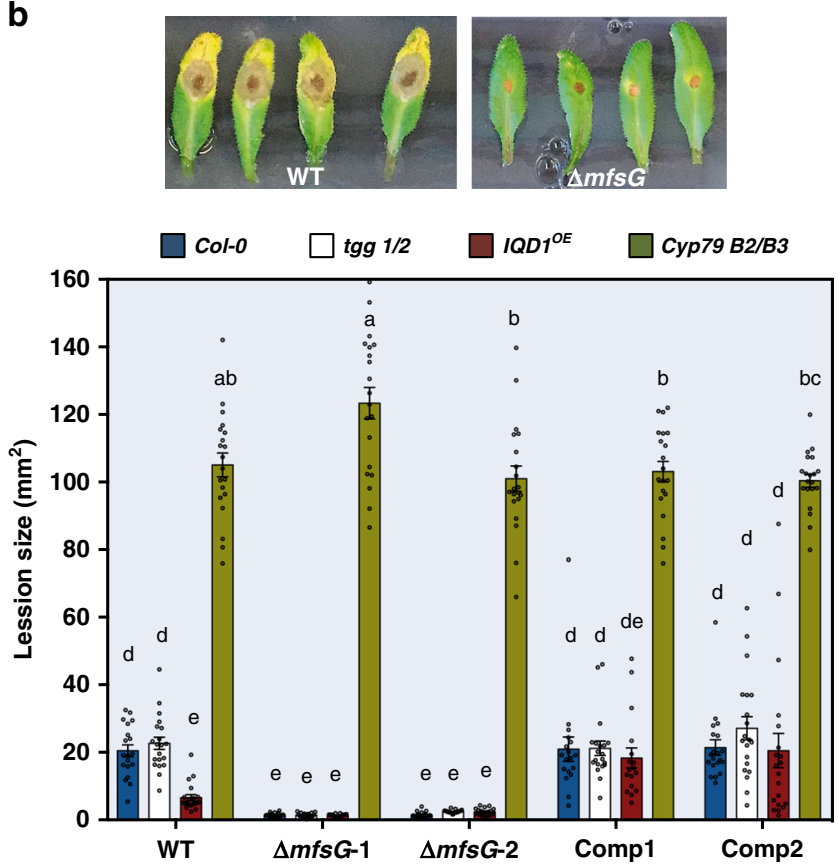

Fig. $4 \mathrm{mfs}$ expression levels and pathogenicity assays on Arabidopsis plants. a $\mathrm{mfs} G$ expression analysis $48 \mathrm{~h}$ after inoculation of wild-type $B$. cinerea on Arabidopsis wild type (WT; Col-0), IQD1OE, cyp79B2/B3 and $\operatorname{tgg} 1 / 2, n=3$. $\mathbf{b}$ Pathogenicity of $B$. cinerea wild type (WT), knockout mutants ( $\triangle m f s G)$, and complemented mutants (Comp) was evaluated on different Arabidopsis genotypes by measuring lesion sizes $72 \mathrm{~h}$ after inoculation with the different $B$. cinerea strains (representative pictures are presented for Col-O Arabidopsis leaves inoculated with WT B05.10 or $\Delta m f s G)$. Average lesion sizes of 17-20 leaves of each genotype are presented together with the standard errors for each average. Different letters above the columns indicate significant difference at $P<0.05$, as determined by Kruskal-Wallis test. Source data are provided as a Source Data file

on the role of MFS transporters as key components in plant-pathogen interactions. In previous study, we showed that A. thaliana mutants with low GS content are more susceptible to $B$. cinerea $^{6,25}$, other works also suggested that $B$. cinerea has variable sensitivity to GSs, as demonstrated by the enhanced susceptibility to $B$. cinerea of cyp79B2/B3 compared with the wild type 6 . Thus, our and others' studies show that ArabidopsisBotrytis interactions are influenced at the metabolic level by variations in toxin production in the host and sensitivity in the pathogen. In the current study, we successfully showed that the MFS transporter $m f s G$ is a crucial component contributing to $B$. cinerea virulence on GS-containing Brassicaceae plants such as A. thaliana. We demonstrated that $m f_{s} G$ expression was upregulated upon exposure to ITCs and on GS-containing plants (Fig. 1

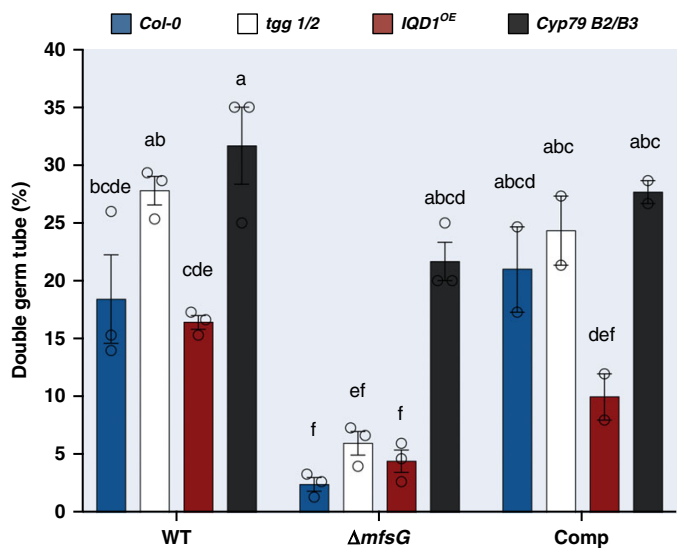

Fig. 5 Germination test of $B$. cinerea $\Delta m f s G$ mutants and complemented mutants. The number of double germ tubes in germinating conidia was evaluated by bright-field microscopy $24 \mathrm{~h}$ after inoculation of Arabidopsis wild type (WT; Col-0), IQD1OE and tgg1/2 with B. cinerea WT, knockout mutants ( $\triangle m f s G)$ and complemented mutants (Comp). Data are displayed as mean values $( \pm S E)$. Different letters above the columns indicate significant difference at $P<0.05$, as determined by Tukey-Kramer HSD test, $n>20$. Source data are provided as a Source Data file

and 4). We found a high correlation between linear mycelium inhibition and relative expression of $m f_{s} G$ in response to different concentrations of BITC (Pearson's $r=0.9958$ ). Resistance to antifungal compounds is commonly regulated at the transcriptional level, as shown by the upregulation of many transporters following exposure to specific toxins. Upregulation of transporters in plant pathogens indicates their role in the excretion of specific and non-specific host toxins as a protective strategy against plant defense compounds. For example, transcriptomic analysis of the Brassicaceae pathogen Alternaria brassicicola exposed to ITCs suggested that protection against Brassicaceae metabolites involves mechanisms aimed at limiting their intracellular accumulation, such as melanin biosynthesis and drug efflux.

Here we showed that $m f s G$ is involved in ITC efflux, as suggested by the excess accumulation of FITC in $\Delta m f_{s} G$ hyphae as compared with the wild type (Fig. 3). Plant pathogens use several modes of action to detoxify phytoalexins and phytoanticipins. General detoxification mechanisms such active efflux is powerful particularly in a broad host range pathogens such as $B$. cinerea. Several reports support our findings that $B$. cinerea uses transporters as a detoxification strategy for toxic compounds and plant secondary metabolites. For example, the $B$. cinerea $A B C$ transporter $B$ catr $B$, involved in camalexin ${ }^{14}$ and resveratrol detoxification, confers pathogenicity on Arabidopsis and grapevine plants, respectively ${ }^{10}$. Moreover, it is also involved in detoxification of antibiotics and fungicides such as fludioxonil ${ }^{37}$ and phenazine ${ }^{11}$. Other transporters, such as BcatrD and Bcmfsl, have also been reported to be involved in azole fungicide transport.

We further demonstrated reduced virulence of $B$. cinerea mutant $\triangle m f_{s} G$ on wild-type $A$. thaliana and enhanced susceptibility of the cyp79B2/B3 mutant compared with Col-0 when inoculated with $\Delta m f_{s} G$ (Fig. 4). Fitness of $\Delta m f_{s} G$ was also reduced in vitro and in planta, as verified by the lower frequency of double germ tubes (Fig. 5 and Supplementary Fig. 3). MFS transporters have been previously implicated in Cercospora nicotianae virulence, causing frog-eye disease on tobacco, where disruption of the CTB4 gene displayed a drastic reduction in cercosporin secretion and a significant reduction in fungal virulence ${ }^{38}$. On the other hand, although the Mycosphaerella graminicola $M f_{s} 1$ confers resistance to azoles and cycloheximide, 


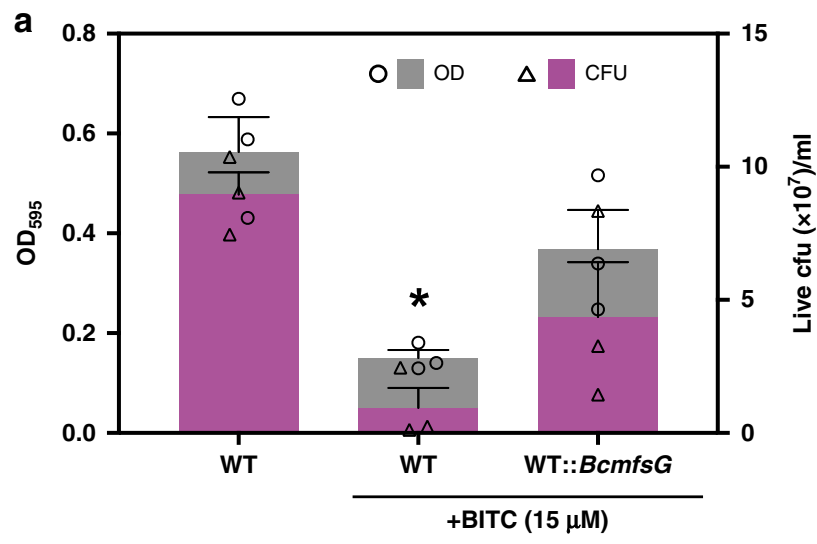

b
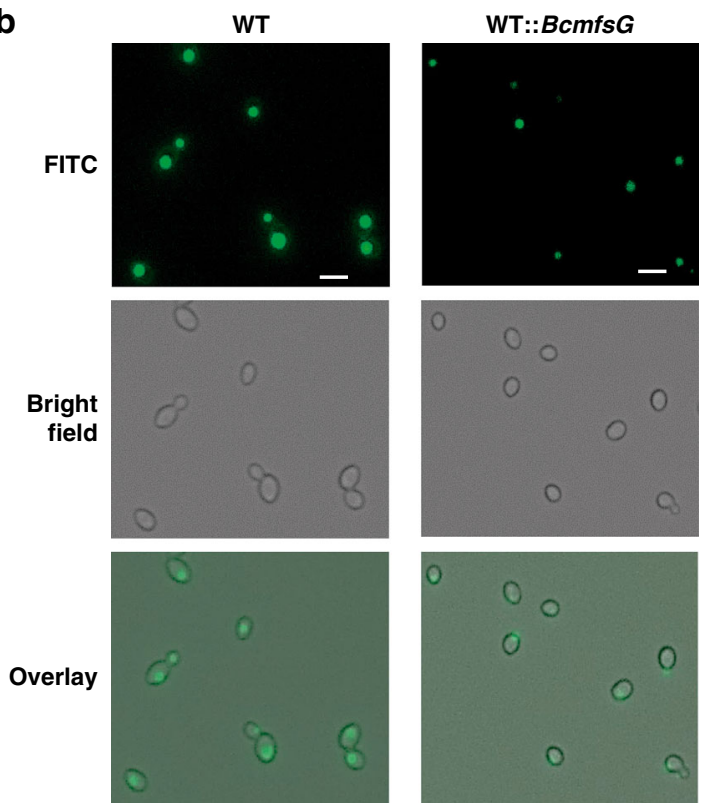

C

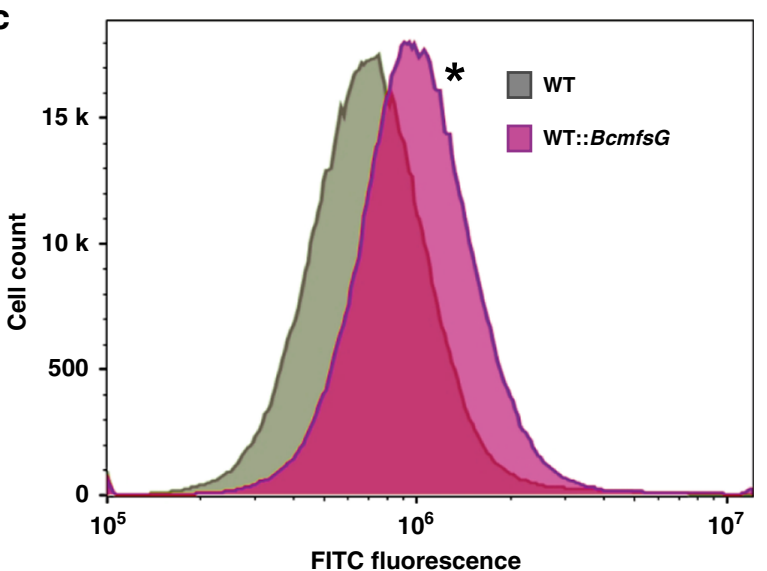

whereas its inactivation abolishes its resistance to fungicides ${ }^{39}$, the virulence on wheat seedlings of $\Delta M g M f s 1$ was not affected ${ }^{40}$.

The slightly reduced virulence of $\Delta m f_{s} G$ mutants on tomato plants (Supplementary Fig. 7) suggests that $m f_{s} G$ might also be involved in the detoxification of other toxic compounds, similar to $B c a t r B, B c a t r D$, and $B c m f s 1$. The simultaneous resistance to a number of unrelated compounds is called multidrug resistance (MDR). It can play a major role in the resistance of plant pathogens, including $B$. cinerea, to agricultural fungicides. Therefore, identification and characterization of drug
Fig. 6 Ectopic expression of $B$. cinerea $m f s G$ and efflux study in yeast. a Saccharomyces cerevisiae wild-type cells (WT), and cells expressing BcmfsG (WT::BcmfsG) were grown with or without $15 \mu \mathrm{M}$ BITC in liquid media. OD and live colony-forming units (cfu) were measured after $48 \mathrm{~h}$. Data are displayed as mean values $( \pm \mathrm{SE})$. Asterisk denotes significant difference by Student's $t$ test $(P<0.001), n=3$. b S. cerevisiae WT and WT:: $B c m f s G$ cells were grown in liquid media with FITC and monitored under a fluorescence microscope $2 \mathrm{~h}$ later. c Fluorescence intensity was measured in WT as compared with WT::Bcmfs cells $2 \mathrm{~h}$ after exposure to FITC using flow cytometry. Asterisk denotes significant difference by Student's $t$ test $(P<0.001), n=100,000$ cells. Scale bars are $10 \mu \mathrm{m}$. Source data are provided as a Source Data file

transporters in plant pathogens is crucial to producing effective management strategies for fungicide resistance.

MDR families of transporters are widely distributed in different types of cells. However, only two are found in all classes of organisms: the $\mathrm{ABC}$ and $\mathrm{MFS}^{41-44}$. In general, MFS transporters are ubiquitous transporters that can only transport small solutes in response to chemiosmotic ion gradients. However, in some cases, MFS transporters are also believed to act as drug transporters using a proton gradient, thus conferring multidrug and multixenobiotic resistance in bacteria, fungi, and more-complex eukaryotes ${ }^{41,42}$. Nevertheless, in general, MFS transporters are more substrate specific compared with $\mathrm{ABC}$ transporters ${ }^{45,46}$. For example, the phenotype of $B$. cinerea MDR2, a field strain that is resistant to several fungicides, is correlated with increased drug-efflux activity via overexpression of the $m f s M 2$ transporter ${ }^{47,48}$. However, using the MDR2 isolate, we could not see any resistance to BITC (Supplementary Fig. 8), supporting our hypothesis of $m f_{s} G$ s specificity for GS-breakdown products. Furthermore, the drug-efflux MFS transporters can also confer MDR in mammalian pathogens; for example, $M d r 1$ from Candida albicans has been reported as a MDR transporter that confers resistance to fungicides such as fluconazole and ketoconazole ${ }^{41,49,50}$. Expression of CaMdr1 confers MDR also to the model yeast $S$. cerevisiae ${ }^{51}$. We also demonstrated that $B$. cinerea $m f_{s} G$ can confer tolerance to BITC in S. cerevisiae yeast cells by providing ITC efflux ability (Fig. 6).

Plant pathogens must protect themselves, especially during infection when they are likely to encounter host defense mechanisms. Therefore, membrane transporters are key elements in toxic compound detoxification. They play a role in counteracting the physiological impact of antimicrobial host defense compounds, as evidenced by the large number of MFS transporters in the genomes of necrotrophic fungal plant pathogens such as Sclerotinia sclerotiorum and B. cinerea: 218,286 , and 282 genes in S. sclerotiorum, B. cinerea T4 and B. cinerea B05.10, respectively. Genome comparison showed that many $B$. cinerea genes, absent in $S$. sclerotiorum, had no orthologues in other fungi, indicating that gene expansion in $B$. cinerea leads to the observed differences between plant-pathogen genomes ${ }^{52}$. There are also variations among the different $B$. cinerea isolates in their response and resistance to GSs and in their transporters' availability $^{25}$ (Fig. 8). BLAST analysis with $m f_{s} G$ transporter revealed two $m f$-like genes in B05.10 that share over $40 \%$ identity with $m f s G$. These two $m f s$-like genes were also found in other $B$. cinerea isolates (T4 and DW1) and in B. cinerea's close relative Sclerotinia sclerotiorum (Fig. 8). Although isolate DW1 had a homolog of $m f_{s} G$, T4 only had the $m f$ s-like homologs (Fig. 8). Docking analysis demonstrated that only $m f s G$ can efficiently bind GS-breakdown products (Table 1 ), whereas $m f$-like proteins have lower probability to bind ITCs (Supplementary Table 2). This may also support the hypothesis of $m f_{s} G$ 's specific involvement in GS detoxification (Table 1 and Fig. 7). B. cinerea T4 and S. sclerotiorum may have evolved other detoxification 
a
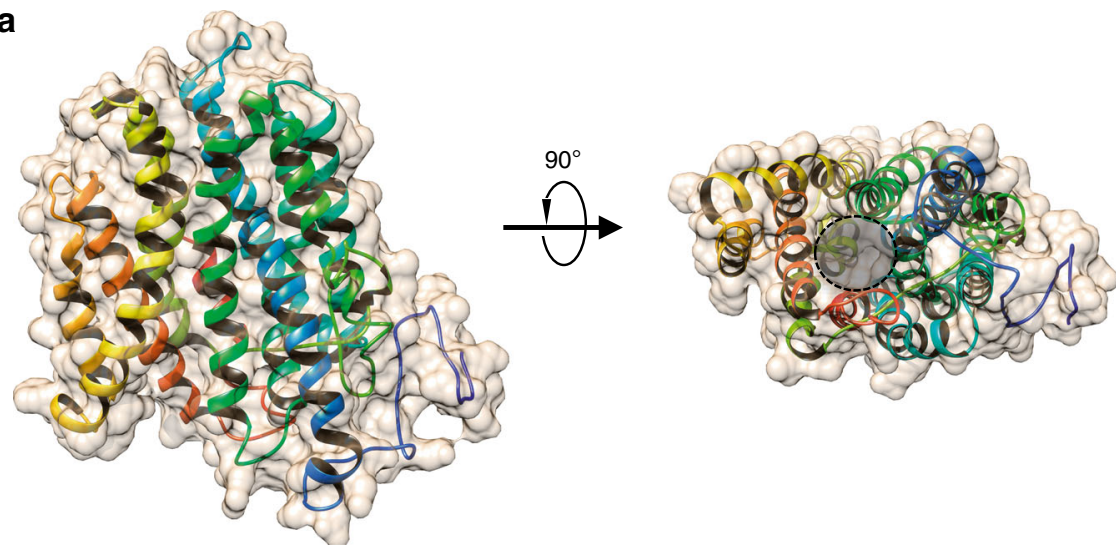

b
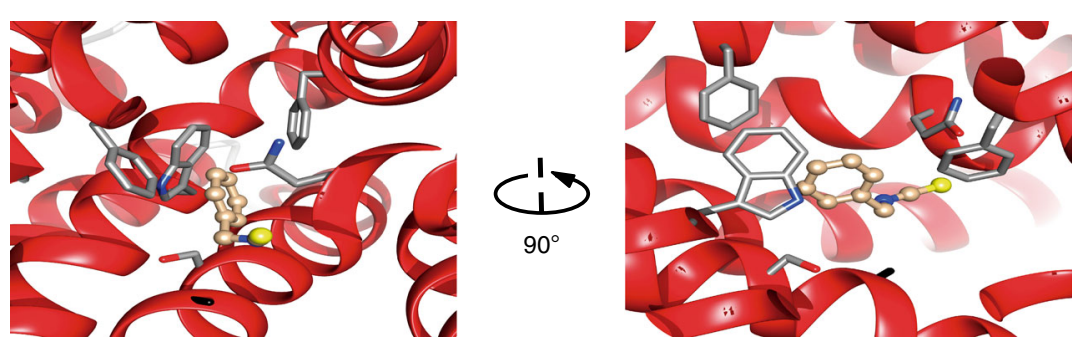

C

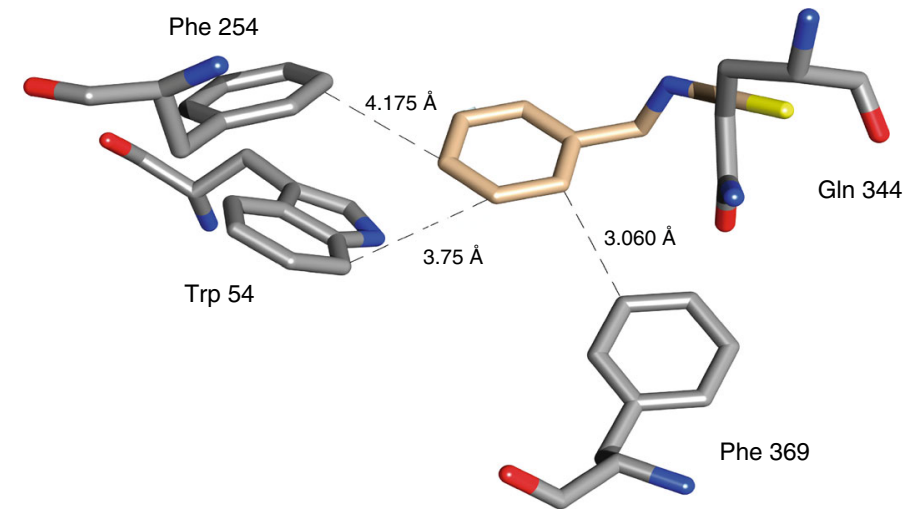

Fig. 7 MFS motif tertiary structure and docking analysis of $B$. cinerea mfsG. a MFS tertiary structure viewed from the side of the membrane, and rotated through 90\%; the conserved 12 transmembrane (TM) $\alpha$-helix fold is arranged into two 6 TM helix bundles forming a cavity (marked with dashed circle) at the interface between them that is accessible to either the cytoplasm or extracellular region, depending on the conformational state. $\mathbf{b}$ Molecular docking between BITC molecule and MFS revealed a putative binding site formed by residues located in the internal channel of MFS. The proposed binding region is formed by the aromatic rings of residues Trp54, Phe254, GIn344, and Phe369. c Distances were measured between the BITC molecule and side chains of these residues, where the biggest distance was $4.175 \AA$ with Phe254

pathways, such as using glutathione S-transferase proteins or other transporters like Alternaria brassicicola ${ }^{53-55}$. It is clear that the natural function of $m f_{s} G$ is to protect $B$. cinerea against aromatic fungitoxic compounds during the pathogenic process. Other studies have postulated that ABC and MFS transporters can also function as effectors or pathogenicity factor secretion, thereby affecting fungal virulence ${ }^{56-59}$. Furthermore, Dos Santos and colleagues' studies on MFS transporters in yeast suggest that these transporters can also provide drug resistance by indirect regulation of the stress response and control of membrane potential. Plant-pathogen transporters may have similar effects.

Finally, we provide evidence of a $B$. cinerea MFS transporter's involvement in GS detoxification and its function as a virulence factor. Further analysis is needed to reveal whether the $B$. cinerea $m f_{s} G$ transporter involved in detoxification of other compounds, and whether it also acts indirectly to provide tolerance to the stress response during plant infection.

\section{Methods}

Plant material and growth conditions. A. thaliana plants: wild-type Columbia $(\mathrm{Col}-0), I Q D 1^{O E}, \operatorname{tgg} 1 / 2$, and $\operatorname{cyp} 79 B 2 / B 3$, were grown at $22^{\circ} \mathrm{C}$ and $60 \%$ relative humidity under fluorescent and incandescent light at a photofluency rate of $\sim 120 \mu \mathrm{mol} \mathrm{m}^{-2} \mathrm{~s}^{-1}$ and a $12 / 12 \mathrm{~h}$ photoperiod. Arabidopsis seeds were stratified on moist soil mix at $4{ }^{\circ} \mathrm{C}$ for 2 days and then transferred to a growth chamber for 4 weeks.

Fungal strains-growth and inoculation of plant material. B. cinerea isolates B05.10 were cultured on potato dextrose agar (PDA, Difco) in a controlledenvironment chamber at $22^{\circ} \mathrm{C}$ in the dark or under illumination with fluorescent and incandescent lights at a photofluency rate of $120 \mu \mathrm{mol} \mathrm{m}{ }^{-2} \mathrm{~s}^{-1}$ and $12 / 12 \mathrm{~h}$ photoperiod. Conidia were harvested from the light-grown culture in sterile distilled water containing $0.001 \%$ (v per v) Triton X-100 (J.T. Baker) and filtered through a $40-\mu \mathrm{m}$ cell strainer to remove remaining hyphae. For inoculation, the conidial suspension was adjusted to $10^{5}$ conidia $\mathrm{ml}^{-1}$ in half-strength filtered $(0.45 \mu \mathrm{m})$ grape juice (100\% pure organic). Detached leaves from 4 -week-old plants were placed on water-agar (1\% w per v) trays and each leaf was inoculated with 4- $\mu \mathrm{l}$ droplets of conidial suspension. Trays were covered with a plastic dome and placed in the growth chamber. At $48 \mathrm{~h}$ post inoculation, leaves were photographed 
a

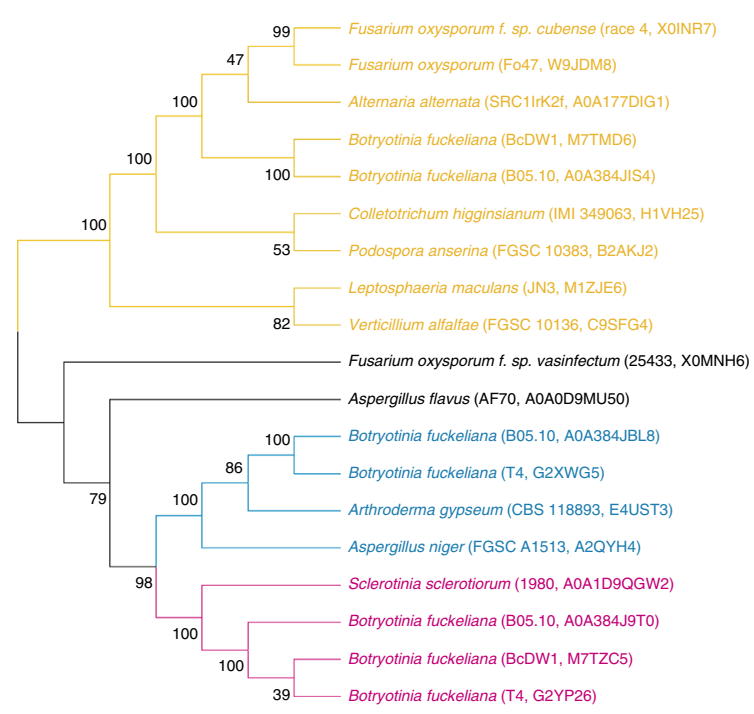

C

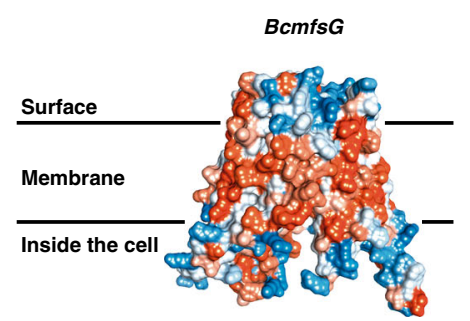

b

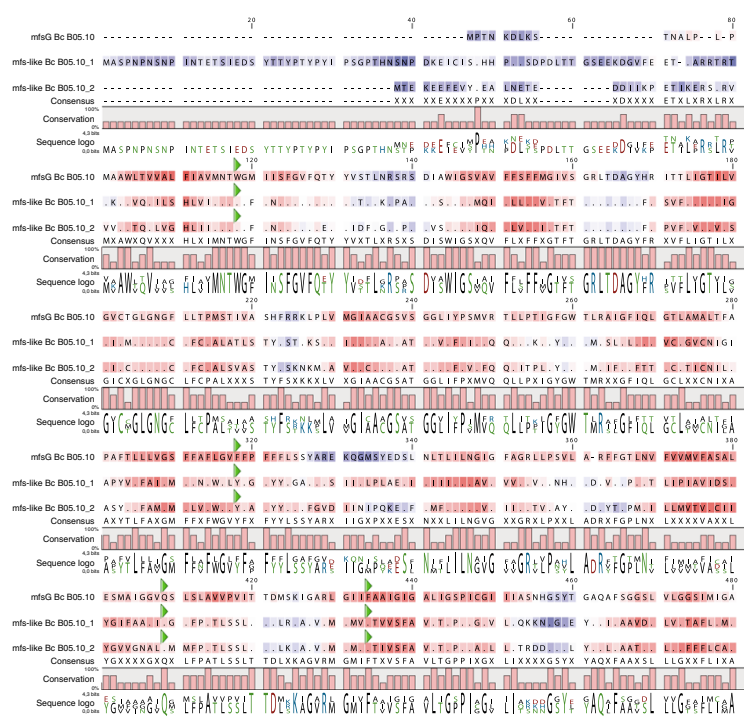

Fig. 8 Phylogenetic and structural analysis of $\mathrm{mfs} G$ and $m f s-l i k e$ proteins. a Phylogenetic analysis was performed using amino-acid sequences of MFS proteins with at least $40 \%$ identity to $\mathrm{mfs}$ from different fungi. All of these sequences were clustered into two clearly differentiated clades. One group contained B05.10 mfs and similar proteins (yellow). The other clade consisted of three subclades: one of them (blue) contained B05.10 mfs-like1 and its homologs, and another (pink) contained B05.10 mfs-like2 and its homologs and a third that do not contain B05.10 homolog (black). Isolate name and protein ID are in parentheses. Bootstrapping (1000 replicates/iterations) was used to generate the phylogenetic tree with the neighbor-joining algorithm in MEGA7. b Alignment of amino-acid sequence of three MFSs from B. cinerea B05.10 ( $\mathrm{mfs}$, mfs-likel, and mfs-like2). Residues located in the proposed binding site to ITCs are highlighted (green triangles). c Three-dimensional structure and hydrophobicity representation of $m f s G, m f s-l i k e 1$, and $m f s-l i k e 2$ from B. cinerea B05.10. d Comparison of three-dimensional structures of $m f s G, m f s-l i k e 1$, and $m f s-l i k e 2$

\section{Table 1 Thermodynamic parameters of molecular docking of ITCs to $\mathbf{m f s} G$}

$\Delta \mathrm{G}^{\mathbf{0}}\left(\mathrm{Kcal} \mathrm{mol}^{-\mathbf{1}}\right)^{\mathrm{a}}$ Full Fitness $\left(\mathrm{Kcal} \mathrm{mol}^{-\mathbf{1}}\right)^{\mathrm{b}}$

BITC
PITC

PITC
PhITC

FITC

5-(Methylsulfanyl)

pentanenitrile

Benzonitrile

Benzyl cyanide

aFree Gibbs energy ${ }^{b}$ Score of docking analysis

and lesion sizes were analyzed using ASSESS 2.0 image analysis software for plant disease quantification (APS Press)

Yeast strain and growth conditions. S. cerevisiae BY4742 (MATa, his $3 \Delta 1$, leu $2 \Delta 0$, lys $2 \Delta 0$, ura3 $\Delta 0$ ) (kindly provided by Dr. Juergen Stolz, Technical University of Munich, Germany) yeast cells were grown in YPD (2\% peptone, $2 \%$ glucose/galactose, $1 \%$ yeast extract, $\mathrm{w}$ per v) or YNB ( $2 \%$ galactose/glucose and $0.67 \%$ yeast nitrogen base, w per v) medium at $30^{\circ} \mathrm{C}$.

ITC inhibition of radial mycelial growth in vitro. The inhibitory effect of ITCs on mycelial growth of B. cinerea (B05.10), $\Delta m f s G$ and the complemented mutants was tested by placing a 6 - $\mathrm{mm}$ diameter plug from 3 -day-old $B$. cinerea colony margins grown in the dark at $22^{\circ} \mathrm{C}$ on a freshly prepared PDA plate containing different concentrations of ITCs. The diameter of radial mycelial growth (D, in $\mathrm{mm}$ ) was recorded at $24 \mathrm{~h}$ for treatments $(\mathrm{Dt})$, and controls $(\mathrm{Dc})$. The fungitoxic effects of ITCs were calculated as percentage of growth inhibition (GI) as: GI $\%)=(D c-$ $D \mathrm{t}) / D \mathrm{c} \times 100$. PITC, BITC and PhITC were obtained from Sigma-Aldrich. Stock solutions of each ITC were prepared in methanol.

RNA extraction, CDNA synthesis, and qRT-PCR. RNA was extracted from $B$. cinerea mycelium after $48 \mathrm{~h}$ growth on PDA supplemented with different ITCs using Plant/Fungi Total RNA Purification Kit (Norgene Biotek Corp). For fungal RNA in planta, total RNA was extracted from $100 \mathrm{mg}$ leaves (12 leaves from three different plants for each treatment) $60 \mathrm{~h}$ post inoculation with $B$. cinerea using TriReagent (Sigma-Aldrich) according to the manufacturer's instructions.

RNA was treated with DNase using the TURBO DNA-Free Kit (Ambion) according to manufacturer's directions. DNA-free RNA $(0.5-1 \mu \mathrm{g})$ was reversetranscribed to cDNA using the High-Capacity cDNA Reverse Transcription Kit (Applied Biosystems) according to the manufacturer's instructions. The cDNA was used as a template in qRT-PCR with Fast SYBR Green Master Mix (Applied Biosystems) and a StepOne real-time PCR machine (Applied Biosystems) according to the manufacturer's protocol. The thermal cycling program was as follows: $94^{\circ} \mathrm{C}$ for $2 \mathrm{~min} ; 40$ cycles of $94^{\circ} \mathrm{C}$ for $15 \mathrm{~s}, 67^{\circ} \mathrm{C}$ for $45 \mathrm{~s}$, and $72^{\circ} \mathrm{C}$ for $1 \mathrm{~min}$; final extension step at $72^{\circ} \mathrm{C}$ for $10 \mathrm{~min}$. Relative fold change of mRNA levels of $m f s G$ (using primers MFS-F and MFS-R), were normalized to B. cinerea actin (BC1G_08198; using primers Actin-F and Actin-R) and calculated by the $2^{-\Delta \Delta C t}$ method using StepOne Plus analysis software version 2.3 (Applied Biosystems). The primer sequences are shown in Supplementary Table 3.

Construction of $\boldsymbol{m} \boldsymbol{f s} \mathbf{G}-\mathbf{k n o c k o u t}$ cassette. The $m f_{s} G$ (BC1G_15286; BCIN_06g00026)-knockout cassette was constructed based on Multiple Gateway 
Three Fragment Vector Construction technology (Invitrogen) ${ }^{60,61}$. The Hyg cassette (2935 bp) was amplified from the pOliHP plasmid using primers attB1hygF and attB2hygR (Supplementary Table S3) 62,63. This fragment was cloned into entry clone plasmid pDONR221 by a BP recombination reaction. PCR fragments of $504 \mathrm{bp}$ were amplified from the $5^{\prime}$ and $3^{\prime}$ regions of $m f s G$, respectively, using primers attB3'F and attB3'R for the $5^{\prime}$ region and primers attB5' $\mathrm{F}$ and attB5'R for the $3^{\prime}$ region (Supplementary Table 3 ). These fragments were individually cloned into entry clone plasmid pDONR P4-P1R and pDONR P2R-P 3, respectively, by BP recombination reaction. After sequencing all three plasmids, a MultiSite Gateway LR recombination reaction was performed between multiple entry clones and the pDEST ${ }^{m} \mathrm{R} 4-\mathrm{R} 3$ vector to generate an expression clone. This final plasmid was named pDEST- $\Delta m f s G$, and was linearized by enzymatic digestion using DraI. The replacement cassette was purified by ethanol precipitation and resuspended in sterile water supplemented with $0.01 \%$ (v per v) Silwet L-77 surfactant (Agri-Turf Supplies) prior to transformation.

Plasmid construction for complementation in B. cinerea. The primers MFSpOliNat1-F and MFSpOliNat1-R (Supplementary Table 3) were used to amplify the full-length wild-type $m f s$ gene from genomic DNA of B. cinerea B05.10. This fragment was cloned into the plasmid pOliHP, replacing the $h p h$ gene, by restriction-free (RF) cloning method, then DpnI-treated and finally transformed into E. coli DH5a, resulting in the plasmid pOliMFS. The nat 1 gene, amplified from the plasmid pNAN-OGG ${ }^{64}$ using the primers NatlpOli-F and Nat1pOli-R (Supplementary Table 3), was cloned into the plasmid pOliMFS, generating pOliMFSNatl which was used for transformation of B. cinerea $\Delta m f s G$ to generate $m f_{s} G$-complementation ${ }^{65}$.

Transformation of B. cinerea. B. cinerea (B05.10) sclerotial transformation was performed. $B$. cinerea sclerotia were collected from mature colonies grown on PDA plates for 10 days at $18^{\circ} \mathrm{C}$. Sclerotia (50-60) were disinfected by three washes with $1 \%$ sodium hypochlorite, followed by three washes with sterilized purified water, and were completely dried prior to transformation on sterile Whatman filter paper in a biological hood. The dried sclerotia were wounded by making a hole in the middle of the sclerotium (without penetrating through) with a sterile needle $(21 \mathrm{G})$, followed by application of $5 \mu \mathrm{l}$ DNA solution of linearized pDEST- $\Delta m f s G$ (a total of $0.5 \mu \mathrm{g}$ ) supplemented with $0.01 \%$ Silwet L-77. Vacuum ( $600 \mathrm{mbar})$ was applied for $10 \mathrm{~min}$, the solution was fully absorbed, and sclerotia and were further dried in a biological hood. Sclerotia were placed on PDA plates $\left(10 \mathrm{ml} \mathrm{plate}^{-1}\right)$ and incubated at $24^{\circ} \mathrm{C}$ for $48 \mathrm{~h}$ of recovery. The growing hyphae were then transferred onto new plates containing PDA supplemented with $25 \mu \mathrm{g} \mathrm{ml}^{-1}$ Hygromycin B (TOKUE). Putative mutants were purified by hyphal tip transfer to new selection plates with increasing hygromycin concentrations of $5 \mu \mathrm{g} \mathrm{ml}^{-1}$ to $40 \mu \mathrm{g} \mathrm{ml}^{-1}$ (each concentration was applied five times). The mutants were grown on PDA supplemented with hygromycin and genomic DNA purification was performed using the MasterPure $^{\mathrm{TM}}$ Yeast DNA Purification Kit (Epicentre) according to the manufacturer's protocol. To verify transformation, we performed PCR analyses on DNA extracted from putative transformants using hygromycin cassette primers Hyg-F and Hyg-R (Supplementary Table 3 and Supplementary Fig. 9).

All PCR analyses were performed in $0.25-\mathrm{ml}$ tubes containing PCR reagent (ReddyMix ${ }^{\oplus}$, Thermo Fisher Scientific Inc.) with 5 pmol of primers, $12.5-25 \mathrm{ng}$ template DNA and sterile purified water to a final volume of $25 \mu \mathrm{l}$. PCR was carried out in an MJ Mini Thermal Cycler (BioRad). Enzyme activation was carried out at $95^{\circ} \mathrm{C}$ for $4 \mathrm{~min}$, followed by denaturation for $30 \mathrm{~s}$ at $95^{\circ} \mathrm{C}$, and annealing at $55^{\circ} \mathrm{C}$ for $40 \mathrm{~s}$, elongation at $72^{\circ} \mathrm{C}$ for $40 \mathrm{~s}$ for $40 \mathrm{cycles}$, and $10 \mathrm{~min}$ of elongation at $72{ }^{\circ} \mathrm{C}$.

For knockout mutant complementation, B. cinerea $\Delta m f s G$ mutants were transformed by conidial electroporation. Fresh spore suspension was prepared as described above (Fungal strains-growth and inoculation of plant material), centrifuged and resuspended in $50 \mathrm{ml}$ of potato dextrose broth (PDB, BD Biosciences). The spore suspension was then incubated for $4 \mathrm{~h}$ at $25^{\circ} \mathrm{C}$ and $100 \mathrm{rpm}$. Germinating conidia were collected by centrifugation at $5000 \mathrm{rpm}$ for $10 \mathrm{~min}$ and resuspended in $20 \mathrm{ml}$ of ice-cold $\mathrm{KC}$ buffer $(0.6 \mathrm{M} \mathrm{KCl}, 50 \mathrm{~mm} \mathrm{CaCl})$; this step was repeated twice to wash the cells. Aliquots of $120 \mu \mathrm{l}\left(1.2 \times 10^{6}\right.$ spores $)$ were placed in pre-chilled $0.2-\mathrm{cm}$ electroporation cuvettes and kept on ice. The conidial suspension was gently mixed with $30 \mu \mathrm{g}$ of linearized plasmid and incubated on ice for $10 \mathrm{~min}$. Transformation was conducted by application of electroporation pulses two pulses at $1.70 \mathrm{kV}, 800 \Omega, 25 \mu \mathrm{F}$ with an interval of $5 \mathrm{~s}$ using an electroporator (Eppendorf 2510 system) ${ }^{66}$. Conidia were then directly resuspended in $1 \mathrm{ml}$ ice-cold KC buffer and placed on ice for $10 \mathrm{~min}$. Conidial suspension was then mixed with $10 \mathrm{ml}$ tempered $\mathrm{SH}$ agar $(1 \mathrm{mM} \mathrm{NaNO}, 0.6 \mathrm{~mm}$ sucrose, $5 \mathrm{~mm}$ Tris $\mathrm{pH}$ 6.5), poured on plates and incubated at $25^{\circ} \mathrm{C}$. After $48 \mathrm{~h}$, the plates were covered with a $10 \mathrm{ml}$ layer of SH agar amended with $50 \mu \mathrm{M} \mathrm{BITC}$ and/ or $40 \mu \mathrm{m}$ nourseothricin (Nat) and the two-layer plates were incubated at $25^{\circ} \mathrm{C}$ for 2 weeks until the transformants grew through the agar. The putative transformants were transferred to PDA plates where the concentration of selection agents was increased progressively to $100 \mu \mathrm{m}$ for BITC and $80 \mu \mathrm{m}$ Nat.

\section{Molecular characterization of $\Delta m f s G$ mutants and complemented mutants.} Putative B. cinerea $\Delta m f s G$ mutants were grown on PDA plates amended with $40 \mu \mathrm{g}$ $\mathrm{ml}^{-1}$ hygromycin. Genomic DNA was extracted using plant/fungi Total DNA Purification Kit (Norgene Biotek Corp.) and RNA was extracted from small amounts of mycelium as described in the RNA extraction section. To confirm the knockout mutation, the expression of $m f s G$ was analyzed by RT-PCR amplifying a 300-bp fragment with primer pairs MFS-F and MFS-R (Supplementary Fig. 9 and Supplementary Table 3). In addition, the presence of $h p h$ gene promoter, POliC, was confirmed in putative mutants amplifying a 552-bp fragment by PCR from genomic DNA with the primers POliC-F and POliC-R (Supplementary Fig. 9 and Supplementary Table 3). B. cinerea $\beta$-tubulin (Bctub2) was used as a control for cDNA and DNA by amplifying a 500-bp fragment with the primers tub2-F and tub2-R (Supplementary Fig. 9 and Supplementary Table 3). PCR amplifications were conducted using with proofreading Phusion High-Fidelity DNA Polymerase (Thermo Fisher Scientific). The amplification conditions consisted of an initial denaturing step at $98^{\circ} \mathrm{C}$ for $2 \mathrm{~min}$, followed by 35 cycles of $94^{\circ} \mathrm{C}$ for $1 \mathrm{~min}, 52^{\circ} \mathrm{C}$ for $1 \mathrm{~min}, 72^{\circ} \mathrm{C}$ for $1 \mathrm{~min}$, and a final elongation step at $72^{\circ} \mathrm{C}$ for $5 \mathrm{~min}$.

Putative complemented mutants were maintained in PDA plates amended with $40 \mu \mathrm{M}$ Nat. To confirm the complementation, the presence of $m f_{s} G$ cassette was screened by PCR as described above. In addition, the presence of Nat resistance gene (Nat1) was confirmed using the primers nat-F/nat-R (Supplementary Fig. 9 and Supplementary Table 3). PCR conditions were as described above.

\section{GI of $\Delta m f s G$ mutants and complemented mutants on BITC. The wild-type} strain B. cinerea B05.10, $\Delta m f_{s} G$ mutants, and complemented mutants were cultured on PDA amended with a range of different concentrations of BITC: 0, 75, $100,150,200 \mu \mathrm{M}$. Mycelial growth was recorded after $72 \mathrm{~h}$ and GI was calculated relative to fungi cultured on PDA alone.

Germination and double germ tube test in planta. Different genotypes of $A$. thaliana were inoculated with a spore suspension $\left(10^{6}\right.$ spore $\left.\mathrm{ml}^{-1}\right)$ of $B$. cinerea wild-type, $\Delta m f s G$ mutants, and complemented mutants. Detached leaves were dipped in spore suspension for $1 \mathrm{~min}$, and placed on water-agar trays (1\%); $24 \mathrm{~h}$ post inoculation, the leaves were cleared in boiling absolute ethanol for $20 \mathrm{~min}$ in a water bath, followed by a final wash in glycerol:lactic acid:water $(1: 1: 1 \mathrm{v}$ per $\mathrm{v})$ overnight. The leaves were then incubated for $2 \mathrm{~min}$ in aniline blue $(0.2 \% \mathrm{w}$ per v) followed by rinsing in distilled water. Finally, germination rate and number of double germ tubes were calculated under a bright-field microscope.

Efflux study in B. cinerea using FITC. B. cinerea isolates were grown on PDA containing $10 \mu \mathrm{m}$ FITC (Sigma-Aldrich) for 4 days at $22^{\circ} \mathrm{C}$ in the dark. Samples of B. cinerea hyphae were analyzed by confocal microscopy (Zeiss LSM-510). Fluorescence inside the cells and in the media was quantified on confocal photomicrographs using Image J software (NIH).

Pathogenicity test of $\Delta \boldsymbol{m} \mathbf{f s} \boldsymbol{G}$ and complemented mutants. The wild-type $B$. cinerea strain B05.10, $\Delta m f_{s} G$ and full-length complemented mutants were screened by plant infection assay ${ }^{25}$. Detached leaves of different transgenic lines of A. thaliana or mutants impaired in GS production were placed on trays of water-agar media (1\%) and inoculated with $4-\mu \mathrm{l}$ droplets of conidial suspension $\left(10^{5}\right.$ spore $\left.\mathrm{ml}^{-1}\right) ; 72 \mathrm{~h}$ post inoculation, lesions, were measured using the image analysis software ASSESS 2.0 for plant disease quantification. Leaves for pathogenicity study were taken from at least 10 different plants with random location in the growth room. All presented data are representative of at least three independent experiments with similar results.

Heterologous expression of $\boldsymbol{B}$. cinerea $\boldsymbol{m} \boldsymbol{f s} \mathbf{G}$ in yeast. The $m f s G$ (BC1G_15286; BCIN_06g00026) open reading frame was amplified from B. cinerea genomic DNA with primers that added restriction sites adjacent to the start and stop codons (BcmfsG-F-EcoRI and BcmfsG-R-XhoI, respectively; Supplementary Table 3). The PCR products were ligated into pGEMT-easy (Promega), sequence-verified, and finally cloned downstream of the galactose-inducible GAL1 promotor of the multicopy vector pYES2 (Invitrogen). pYES2-BcmfsG was used to transform $S$. cerevisiae yeast strain BY4742 by the lithium acetate procedure ${ }^{67}$. Yeast cells were grown in YPD (glucose) medium till reaches to mid-log phase. Cells were than harvested by centrifugation and washed with TE $(10 \mathrm{~mm}$ Tris- $\mathrm{HCl}(\mathrm{pH} 8.0)$ and $1.0 \mathrm{~mm}$ EDTA) and finally suspended in TE to the final concentration of $2 \times 10^{8}$ cells $\mathrm{ml}^{-1}$. Suspended cells were mixed with an equal volume $(0.5 \mathrm{ml})$ of $0.2 \mathrm{M}$ metal ions (LiAc) to final volume of $1 \mathrm{ml}$ and incubated for $1 \mathrm{~h}$ at $30^{\circ} \mathrm{C}$ with shaking $(140 \mathrm{rpm})$. In total, $100 \mu \mathrm{l}$ of this mixed suspension incubated with $15 \mu \mathrm{l}$ of a plasmid DNA solution $\left(670 \mu \mathrm{g} \mathrm{ml}^{-1}\right)$ at $30^{\circ} \mathrm{C}$ for $30 \mathrm{~min}$. An equal volume of $70 \%$ PEG 4000 dissolved in water was mix thoroughly with the cells and incubated at $30^{\circ} \mathrm{C}$ for $1 \mathrm{~h}$, followed by $5 \mathrm{~min}$ incubation at $42^{\circ} \mathrm{C}$. Cells were immediately cooled to room temperature. Washed twice with water and suspended to $1 \mathrm{ml}$ of water. Selection of transformants was carried out on YNB minimal medium lacking uracyl (auxotrophic selection) and amended with thiamine to prevent possible toxic effects of plasmid expression.

Yeast GI by BITC. The growth-inhibitory effect of BITC was tested using different strains of yeast: BY4742 (WT) and BY4742::BcmfsG (WT::BcmfsG). Strains were grown in liquid and solid media. The yeast strains were grown in YPD medium containing galactose as a carbon source (Sigma-Aldrich) for $5 \mathrm{~h}$ (optical density at $595 \mathrm{~nm}\left[\mathrm{OD}_{595}\right]=0.5$ ). The yeast strains were diluted and $15 \mu \mathrm{M}$ BITC was added 
to the minimal synthetic media for yeast ( $\mathrm{YNB}+$ galactose). The yeast cells were grown at $30^{\circ} \mathrm{C}$ in a plate reader (TECAN, Infinite F200) and the $\mathrm{OD}_{595}$ was recorded (four reads per well in triplicate per sample) and registered for every well (48-well plate, $300 \mu \mathrm{l}$ per well) every hour after shaking. In addition, samples were taken at different time points; the yeast were diluted and cultured on YPD (glucose) agar plates at $30^{\circ} \mathrm{C}$. The colonies were counted and correlated to the results of the $\mathrm{OD}$ readings. The yeast cells were grown at $30^{\circ} \mathrm{C}$ for 2 days before colony counting and the results were analyzed.

FITC efflux in yeast. Photomicrographic and quantitative FITC-efflux assays were performed as described by Preston et al. ${ }^{68}$ with the following adaptations. The yeast strains were grown in YPD medium containing galactose (Sigma-Aldrich) as a carbon source under constant agitation $(150 \mathrm{rpm})$ for $5-8 \mathrm{~h}$ to an $\mathrm{OD}_{595}$ of 0.5 . FITC $\left(10 \mu \mathrm{g} \mathrm{ml}^{-1}\right)$ was added along with $50 \mathrm{~mm}$ sodium citrate and cultures were incubated at $37^{\circ} \mathrm{C}$ for $30 \mathrm{~min}$ in the dark. The yeast cells where then washed three times with minimal synthetic medium YNB (galactose) and incubated at $30^{\circ} \mathrm{C}$. Samples were taken after $2 \mathrm{~h}$ for fluorescence flow cytometry analysis (CytoFlex, Beckman) and for microscopy (EVOS FL AUTO, Thermo Fisher). For flow cytometry analysis no sorting or gating were done, $100 \%$ of the cells showed normal SSC-A/FSC-A distribution (Supplementary Fig. 10) and were included in the analysis using FlowJo software.

Protein modeling and molecular docking. The "Protter server [http://wlab.ethz. $\mathrm{ch} /$ protter/start/]" was used to depict the B. cinerea $m f s G$-encoded protein's secondary structure. "Phyre2 workspace [www.sbg.bio.ic.ac.uk/phyre2]" was used for automated protein tertiary structure homology modeling of this protein ${ }^{32}$. To identify potential binding sites of BITC (PubChem ID: 2346), PITC (PubChem ID: 69403), PhITC (PubChem ID: 7673), FITC (PubChem ID: 113298), 5-(Methylsulfanyl)pentanenitrile (PubChem ID: 93320), Benzonitrile (PubChem ID: 7505), and Benzyl cyanide (PubChem ID: 8794) to the $m f_{s} G$-encoded protein, automated molecular docking and thermodynamic analysis were performed using the webbased "SwissDock program [www.swissdock.ch/docking]" 69 . SwissDock predicts the possible molecular interactions between a target protein and small molecule is based on the docking algorithm EADock DSS ${ }^{70}$. The docking was performed using the "Accurate" parameter at otherwise default parameters, with no region of interest defined (blind docking). Binding energies are estimated by using CHARMM (Chemistry at HARvard Macromolecular Mechanics), a molecular simulation program implemented within SwissDock software, and the most favorable energies are evaluated by FACTS (Fast Analytical Continuum Treatment of Solvation). Finally, the results on energies are scored and ranked by full fitness $\left(\mathrm{kcal} \mathrm{mol}^{-1}\right)$ and the spontaneous binding is exhibited by the estimated Gibbs free energy $\Delta \mathrm{G}\left(\mathrm{kcal} \mathrm{mol}^{-1}\right)$. The negative values of $\Delta \mathrm{G}$ support the assertion that the binding process is highly spontaneous. Modeling and docking results were visualized using UCSF Chimera v1.8 software.

Statistical analysis. When data were normally distributed and sample variances were equal, $t$ tests were performed. In all other cases, Mann-Whitney Rank Sum test was performed. For multiple comparisons, one-way analysis of variance (ANOVA) was performed when the equal variance test was passed. In all other cases, one-way ANOVA on ranks was performed (Kruskal-Wallis or Tukey-Kramer honest significant difference test). Significance was accepted at $P<0.05$.

Reporting summary. Further information on research design is available in the Nature Research Reporting Summary linked to this article.

\section{Data availability}

Data underlying Figs. 1, 2, 3b, 4, 5, 6a, 6c and Supplementary Figs. 2, 3b, 4, 7, 8, and 9 are provided as Source Data files. All other data are available from the corresponding author upon reasonable requests. $B c m f s G$ accession number at NCBI is BCIN_06g00026 (old locus tag is BC1G_15286).

Received: 25 September 2018 Accepted: 4 June 2019

Published online: 28 June 2019

\section{References}

1. Ahuja, I., Kissen, R. \& Bones, A. M. Phytoalexins in defense against pathogens. Trends Plant Sci. 17, 73-90 (2012).

2. Arbona, V. \& Gómez-Cadenas, A. Metabolomics of disease resistance in crops. Curr. Issues Mol. Biol. 19, 13-30 (2016).

3. Smith, D. A. \& Banks, S. W. Formation and biological properties of isoflavonoid phytoalexins. Prog. Clin. Biol. Res 213, 113-124 (1986).

4. VanEtten, H. D., Mansfield, J. W., Bailey, J. A. \& Farmer, E. E. Two classes of plant antibiotics: phytoalexins versus "Phytoanticipins". Plant Cell 6, 1191-1192 (1994).
5. Morrissey, J. P. \& Osbourn, A. E. Fungal resistance to plant antibiotics as a mechanism of pathogenesis. Microbiol. Mol. Biol. Rev. 63, 708-724 (1999).

6. Kliebenstein, D. Secondery metabolites and plant/environment interactions: a view through arabidopsis thaliana tinged glasses. Plant Cell Environ. 27, 675-684 (2004).

7. Vanetten, H. D., Matthews, D. E. \& Matthews, P. S. Phytoalexin detoxification: importance for pathogenicity and practical implications. Annu. Rev. Phytopathol. 27, 143-164 (1989).

8. Pedras, M. S. \& Ahiahonu, P. W. Metabolism and detoxification of phytoalexins and analogs by phytopathogenic fungi. Phytochemistry 66, 391-411 (2005).

9. Pedras, M. S. \& Ahiahonu, P. W. Probing the phytopathogenic stem rot fungus with phytoalexins and analogues: unprecedented glucosylation of camalexin and 6-methoxycamalexin. Bioorg. Med. Chem. 10, 3307-3312 (2002).

10. Schoonbeek, H., Del Sorbo, G. \& De Waard, M. A. The ABC transporter BcatrB affects the sensitivity of Botrytis cinerea to the phytoalexin resveratrol and the fungicide fenpiclonil. Mol. Plant Microbe Interact. 14, 562-571 (2001).

11. Schoonbeek, H. J., Raaijmakers, J. M. \& De Waard, M. A. Fungal ABC transporters and microbial interactions in natural environments. Mol. Plant Microbe Interact. 15, 1165-1172 (2002).

12. Del Sorbo, G., Schoonbeek, H. \& De Waard, M. A. Fungal transporters involved in efflux of natural toxic compounds and fungicides. Fungal Genet. Biol. 30, 1-15 (2000).

13. Rogers, B. et al. The pleitropic drug $\mathrm{ABC}$ transporters from Saccharomyces cerevisiae. J. Mol. Microbiol. Biotechnol. 3, 207-214 (2001).

14. Stefanato, F. L. et al. The ABC transporter BcatrB from Botrytis cinerea exports camalexin and is a virulence factor on Arabidopsis thaliana. Plant $J$. 58, 499-510 (2009).

15. Urban, M., Bhargava, T. \& Hamer, J. E. An ATP-driven efflux pump is a novel pathogenicity factor in rice blast disease. EMBO J. 18, 512-521 (1999)

16. Sun, C. B., Suresh, A., Deng, Y. Z. \& Naqvi, N. I. A multidrug resistance transporter in Magnaporthe is required for host penetration and for survival during oxidative stress. Plant Cell 18, 3686-3705 (2006).

17. Gupta, A. \& Chattoo, B. B. Functional analysis of a novel ABC transporter ABC4 from Magnaporthe grisea. FEMS Microbiol. Lett. 278, 22-28 (2008).

18. Fleissner, A., Sopalla, C. \& Weltring, K. M. An ATP-binding cassette multidrug-resistance transporter is necessary for tolerance of Gibberella pulicaris to phytoalexins and virulence on potato tubers. Mol. Plant Microbe Interact. 15, 102-108 (2002).

19. Stergiopoulos, I., van Nistelrooy, J. G., Kema, G. H. \& De Waard, M. A. Multiple mechanisms account for variation in base-line sensitivity to azole fungicides in field isolates of Mycosphaerella graminicola. Pest Manag Sci. 59, 1333-1343 (2003)

20. Higgins, C. F. ABC transporters: from microorganisms to man. Annu Rev. Cell Biol. 8, 67-113 (1992).

21. Gulshan, K. \& Moye-Rowley, W. S. Multidrug resistance in fungi. Eukaryot. Cell 6, 1933-1942 (2007).

22. Sanguinetti, M. et al. Role of AFR1, an ABC transporter-encoding gene, in the in vivo response to fluconazole and virulence of Cryptococcus neoformans. Infect. Immun. 74, 1352-1359 (2006).

23. van Veen, H. W. \& Konings, W. N. Structure and function of multidrug transporters. Adv. Exp. Med Biol. 456, 145-158 (1998).

24. Webber, M. A. \& Piddock, L. J. The importance of efflux pumps in bacterial antibiotic resistance. J. Antimicrob. Chemother. 51, 9-11 (2003).

25. Buxdorf, K., Yaffe, H., Barda, O. \& Levy, M. The effects of glucosinolates and their breakdown products on necrotrophic fungi. PLoS ONE 8, e70771 (2013).

26. Fillinger, S., and Yigal Elad, Botrytis: the fungus, the pathogen and its management in agricultural systems. (Cham: Springer International Publishing, 2016).

27. Elad, Y., Williamson, B., Tudzynski, B. \& Delen, N. Botrytis: biology, pathology and control. (Springer, Dordrecht, The Netherlands; 2007).

28. Leroux, P. et al. Mechanisms of resistance to fungicides in field strains of Botrytis cinerea. Pest Manag. Sci. 58, 876-888 (2002).

29. Levy, M., Wang, Q., Kaspi, R., Parrella, M. P. \& Abel, S. Arabidopsis IQD1, a novel calmodulin-binding nuclear protein, stimulates glucosinolate accumulation and plant defense. Plant J. 43, 79-96 (2005).

30. Zhao, Y. et al. Trp-dependent auxin biosynthesis in Arabidopsis: involvement of cytochrome P450s CYP79B2 and CYP79B3. Genes Dev. 16, 3100-3112 (2002).

31. Barth, C. \& Jander, G. Arabidopsis myrosinases TGG1 and TGG2 have redundant function in glucosinolate breakdown and insect defense. Plant J. 46, 549-562 (2006)

32. Kelley, L. A., Mezulis, S., Yates, C. M., Wass, M. N. \& Sternberg, M. J. E. The Phyre2 web portal for protein modeling, prediction and analysis. Nat. Protoc. 10, 845 (2015). 
33. Van Kan, J. A. et al. A gapless genome sequence of the fungus Botrytis cinerea. Mol. Plant Pathol. 18, 75-89 (2017).

34. DeRisi, J. et al. Genome microarray analysis of transcriptional activation in multidrug resistance yeast mutants. FEBS Lett. 470, 156-160 (2000).

35. Coleman, J. J. \& Mylonakis, E. Efflux in fungi: la pièce de résistance. PLoS Pathog. 5, e1000486 (2009).

36. Báez-Flores, M. E. et al. Differentially expressed cDNAs in Alternaria alternata treated with 2-propenyl isothiocyanate. Microbiol. Res. 166, 566-577 (2011).

37. Vermeulen, T., Schoonbeek, H. \& De Waard, M. A. The ABC transporter BcatrB from Botrytis cinereais a determinant of the activity of the phenylpyrrole fungicide fludioxonil. Pest Manag. Sci. 57, 393-402 (2001).

38. Choquer, M., Lee, M. H., Bau, H. J. \& Chung, K. R. Deletion of a MFS transporter-like gene in Cercospora nicotianae reduces cercosporin toxin accumulation and fungal virulence. FEBS Lett. 581, 489-494 (2007).

39. Omrane, S. et al. Fungicide efflux and the MgMFS1 transporter contribute to the multidrug resistance phenotype in Zymoseptoria tritici field isolates. Environ. Microbiol. 17, 2805-2823 (2015).

40. Roohparvar, R., De Waard, M. A., Kema, G. H. \& Zwiers, L. H. MgMfs1, a major facilitator superfamily transporter from the fungal wheat pathogen Mycosphaerella graminicola, is a strong protectant against natural toxic compounds and fungicides. Fungal Genet. Biol. 44, 378-388 (2007).

41. Dos Santos, S. C., Teixeira, M. C., Dias, P. J. \& Sá-Correia, I. MFS transporters required for multidrug/multixenobiotic (MD/MX) resistance in the model yeast: understanding their physiological function through post-genomic approaches. Front. Physiol. 5, 180 (2014).

42. Du, D. et al. Multidrug efflux pumps: structure, function and regulation. Nat. Rev. Microbiol. 16, 523-539 (2018).

43. Higgins, C. F. Multiple molecular mechanisms for multidrug resistance transporters. Nature 446, 749-757 (2007).

44. Kovalchuk, A. \& Driessen, A. J. Phylogenetic analysis of fungal ABC transporters. BMC Genomics 11, 177 (2010).

45. Kaback, H. R. A chemiosmotic mechanism of symport. Proc. Natl Acad. Sci. USA 112, 1259-1264 (2015).

46. Zhang, X. C., Zhao, Y., Heng, J. \& Jiang, D. Energy coupling mechanisms of MFS transporters. Protein Sci. 24, 1560-1579 (2015).

47. Kretschmer, M. et al. Fungicide-driven evolution and molecular basis of multidrug resistance in field populations of the grey mould fungus Botrytis cinerea. PLoS Pathog. 5, e1000696 (2009).

48. Sang, H. et al. A pleiotropic drug resistance transporter is involved in reduced sensitivity to multiple fungicide classes in Sclerotinia homoeocarpa (F.T. Bennett). Mol. Plant Pathol. 16, 251-261 (2015).

49. Hiller, D., Sanglard, D. \& Morschhäuser, J. Overexpression of the MDR1 gene is sufficient to confer increased resistance to toxic compounds in Candida albicans. Antimicrob. Agents Chemother. 50, 1365-1371 (2006).

50. Costa, C., Dias, P. J., Sá-Correia, I. \& Teixeira, M. C. MFS multidrug transporters in pathogenic fungi: do they have real clinical impact? Front. Physiol. 5, 197 (2014).

51. Mukhopadhyay, K., Kohli, A. \& Prasad, R. Drug susceptibilities of yeast cells are affected by membrane lipid composition. Antimicrob. Agents Chemother. 46, 3695-3705 (2002).

52. Amselem, J. et al. Genomic analysis of the necrotrophic fungal pathogens Sclerotinia sclerotiorum and Botrytis cinerea. PLoS Genet. 7, e1002230 (2011).

53. Sellam, A., Dongo, A., Guillemette, T., Hudhomme, P. \& Simoneau, P. Transcriptional responses to exposure to the brassicaceous defence metabolites camalexin and allyl-isothiocyanate in the necrotrophic fungus Alternaria brassicicola. Mol. Plant Pathol. 8, 195-208 (2007).

54. van Kan, J. A. Licensed to kill: the lifestyle of a necrotrophic plant pathogen. Trends Plant Sci. 11, 247-253 (2006).

55. Sellam, A., Poupard, P. \& Simoneau, P. Molecular cloning of AbGst1 encoding a glutathione transferase differentially expressed during exposure of Alternaria brassicicola to isothiocyanates. FEMS Microbiol Lett. 258, 241-249 (2006).

56. de Waard, M. A. et al. Impact of fungal drug transporters on fungicide sensitivity, multidrug resistance and virulence. Pest Manag. Sci. 62, 195-207 (2006).

57. Hayashi, K., Schoonbeek, H. J. \& De Waard, M. A. Bcmfs1, a novel major facilitator superfamily transporter from Botrytis cinerea, provides tolerance towards the natural toxic compounds camptothecin and cercosporin and towards fungicides. Appl Environ. Microbiol. 68, 4996-5004 (2002).

58. Hayashi, K., Schoonbeek, H. J. \& De Waard, M. A. Modulators of membrane drug transporters potentiate the activity of the DMI fungicide oxpoconazole against Botrytis cinerea. Pest Manag. Sci. 59, 294-302 (2003).

59. Stergiopoulos, I., Gielkens, M. M., Goodall, S. D., Venema, K. \& De Waard, M. A. Molecular cloning and characterisation of three new ATP-binding cassette transporter genes from the wheat pathogen Mycosphaerella graminicola. Gene 289, 141-149 (2002).
60. Shafran, H., Miyara, I., Eshed, R., Prusky, D. \& Sherman, A. Development of new tools for studying gene function in fungi based on the Gateway system. Fungal Genet. Biol. 45, 1147-1154 (2008).

61. Ish-Shalom, S., Gafni, A., Lichter, A. \& Levy, M. Transformation of Botrytis cinerea by direct hyphal blasting or by wound-mediated transformation of sclerotia. BMC Microbiol. 11, 266 (2011).

62. Rolke, Y. et al. Functional analysis of $\mathrm{H}(2) \mathrm{O}(2)$-generating systems in Botrytis cinerea: the major $\mathrm{Cu}-\mathrm{Zn}$-superoxide dismutase (BCSOD1) contributes to virulence on French bean, whereas a glucose oxidase (BCGOD1) is dispensable. Mol. Plant Pathol. 5, 17-27 (2004).

63. van den Ent, F. \& Löwe, J. RF cloning: a restriction-free method for inserting target genes into plasmids. J. Biochem Biophys. Methods 67, 67-74 (2006).

64. Schumacher, J. Tools for Botrytis cinerea: New expression vectors make the gray mold fungus more accessible to cell biology approaches. Fungal Genet. Biol. 49, 483-497 (2012).

65. Hamada, W., Reignault, P., Bompeix, G. \& Boccara, M. Transformation of Botrytis cinerea with the hygromycin B resistance gene, hph. Curr. Genet. 26, 251-255 (1994).

66. Vela-Corcía, D., Romero, D., Torés, J. A., De Vicente, A. \& Pérez-García, A. Transient transformation of Podosphaera xanthii by electroporation of conidia. BMC Microbiol. 15, 20 (2015).

67. Kawai, S., Hashimoto, W. \& Murata, K. Transformation of Saccharomyces cerevisiae and other fungi: methods and possible underlying mechanism. Bioeng. Bugs 1, 395-403 (2010).

68. Preston, R. A., Murphy, R. F. \& Jones, E. W. Apparent endocytosis of fluorescein isothiocyanate-conjugated dextran by Saccharomyces cerevisiae reflects uptake of low molecular weight impurities, not dextran. J. Cell Biol. 105, 1981-1987 (1987).

69. Grosdidier, A., Zoete, V. \& Michielin, O. SwissDock, a protein-small molecule docking web service based on EADock DSS. Nucleic Acids Res. 39, W270-W277 (2011)

70. Grosdidier, A., Zoete, V. \& Michielin, O. EADock: docking of small molecules into protein active sites with a multiobjective evolutionary optimization. Proteins 67, 1010-1025 (2007).

\section{Acknowledgements}

We want to thank Dr. Schlesinger S. for her help with flow cytometry analysis and to Dr. Zelinger-Reichert E. for her help with microscopy.

\section{Author contributions}

D.V.C, D.A.S, A.D.B and M.L. designed the experiments. D.V.C, A.D.B, D.A.S, N.R. and O.B. performed the experiments. D.V.C, A.D.B., and M.L. analyzed the results and wrote the manuscript. All authors read and approved the final manuscript.

\section{Additional information}

Supplementary Information accompanies this paper at https://doi.org/10.1038/s41467 019-10860-3.

Competing interests: The authors declare no competing interests.

Reprints and permission information is available online at http://npg.nature.com/ reprintsandpermissions/

Peer review information: Nature Communications thanks Sabine Fillinger, Philippe Simoneau and other anonymous reviewer(s) for their contribution to the peer review of this work.

Publisher's note: Springer Nature remains neutral with regard to jurisdictional claims in published maps and institutional affiliations.

Open Access This article is licensed under a Creative Commons Attribution 4.0 International License, which permits use, sharing, adaptation, distribution and reproduction in any medium or format, as long as you give appropriate credit to the original author(s) and the source, provide a link to the Creative Commons license, and indicate if changes were made. The images or other third party material in this article are included in the article's Creative Commons license, unless indicated otherwise in a credit line to the material. If material is not included in the article's Creative Commons license and your intended use is not permitted by statutory regulation or exceeds the permitted use, you will need to obtain permission directly from the copyright holder. To view a copy of this license, visit http://creativecommons.org/ licenses/by/4.0/.

(C) The Author(s) 2019 